\title{
Molecular and Functional MRI of the Tumor Microenvironment
}

In malignant tumors, cancer cells are embedded within a complex microenvironment consisting of the extracellular matrix (ECM), blood and lymphatic vessels, infiltrating leukocytes, fibroblasts, and other stromal cells. This microenvironment is characterized by abnormal physiologic conditions such as hypoxia and acidic extracellular $\mathrm{pH}$, generated largely by the chaotic tumor vasculature and lack of well-established lymphatics. Cancer cells can significantly contribute to this abnormal tumor microenvironment (TME) through increased glycolysis, upregulation of inflammatory pathways, and the secretion of proteolytic enzymes (1). Because several characteristics of the cancer cell are influenced by the interactions between cancer cells and the TME, the TME is increasingly occupying center stage in cancer etiology, progression, and response to treatment. Because of the remarkable ability of cancer cells to adapt and survive, finding effective treatments against cancer depends on identifying and targeting pathways critical for the survival of the cancer cells. Understanding and characterizing the TME provide unique opportunities to identify novel targets for cancer therapy.

Multimodality and multiparametric molecular and functional imaging provide opportunities for imaging the TME and its interactions with cancer cells. Magnetic resonance imaging (MRI) and magnetic resonance spectroscopy (MRS) have traditionally been used to characterize functional tumor parameters such as $\mathrm{pH}$, vascularization, and metabolism (2). The abundant water signal is typically used to obtain MR images with high spatial resolution. The magnetization signal is characterized by 2 rate constants, the spin-lattice (longitudinal relaxation time), or $\mathrm{T}_{1}$, and the spin-spin (transverse relaxation time), or $\mathrm{T}_{2}$. Most contrast agents used in MRI generate contrast or are detected by the changes they induce in the $T_{1}$ or $T_{2}$ of water. $T_{1}$ contrast agents enhance the signal in a $\mathrm{T}_{1}$-weighted image, whereas $\mathrm{T}_{2}$ contrast agents result in a loss of signal in a $\mathrm{T}_{2}$-weighted image. MRS distinguishes a particular nucleus with respect to its environment in the molecule because the resonance frequency of a particular nucleus is dependent on its molecular structure. In MR spectroscopic imaging (MRSI), the chem-

\footnotetext{
Received Nov. 29, 2007; revision accepted Mar. 11, 2008.

For correspondence or reprints contact: Zaver M. Bhujwalla, Department of Radiology, Johns Hopkins University School of Medicine, Room 208C, Traylor Building, 720 Rutland Ave., Baltimore, MD 21205.

E-mail: zaver@mri.jhu.edu

COPYRIGHT @ 2008 by the Society of Nuclear Medicine, Inc

DOI: $10.2967 /$ jnumed.107.043349
}

ical information is spatially encoded to obtain images of metabolites or exogenous substances or drugs. A major advantage of MRI techniques is that they can be translated from bench to bedside with relative ease, allowing repeated acquisition of spatial and temporal information. In this review, we have focused on some uses and advances of molecular and functional MRI techniques to image the TME.

\section{IMAGING OF ENZYME ACTIVITY AND ECM INTEGRITY}

The ability of cancer cells to invade tissue and metastasize to other organs represents two of the most lethal phenotypic traits of cancer. Cancer cells and stromal cells secrete proteolytic enzymes, several of which are secreted as inactive proforms that become activated by components in the ECM (1). The ability to noninvasively image the activity of degradative enzymes would be useful to characterize the invasiveness of the tumor and to follow the action of proteolytic enzyme inhibitors for therapy $(3,4)$. Recent developments in MRI detection of proteolytic enzymes include the detection of transglutaminases and hyaluronidase $(3,4)$. Transglutaminases mediate tissue remodeling by catalyzing covalent cross-links between proteins of the ECM (3). A lowmolecular-weight reporter was recently developed for MRI detection by using a peptide with a specific recognition sequence for transglutaminase linked to gadolinium-diethylenetriaminepentaacetate (DTPA). Transglutaminase activity generated MRI contrast by altering the spin-lattice relaxation rate $\left(1 / \mathrm{T}_{1}\right)$ of water molecules in contact with the contrast agent (3). A similar mechanism was exploited to detect hyaluronidase activity. Hyaluronan, a major component of the ECM, is antiangiogenic, but its degradation by hyaluronidases produces factors that stimulate endothelial cell proliferation and promote neovascularization (4). Secretion of hyaluronidase by cancer cells may contribute to their invasiveness (4). Hyaluronidase activity was detected in cells and in an ovarian cancer xenograft model by changes induced by the contrast agent in the spin-lattice and spinspin relaxation rate $\left(1 / \mathrm{T}_{2}\right)$ of water $(4)$.

Characterizing the movement of macromolecules through the ECM provides a measure of its integrity. Because proteolytic enzymes degrade the ECM, its integrity or porosity can be used to characterize the invasiveness of the tumor and potentially design treatments that may alter ECM integrity for improved drug delivery to the tumor interstitium. MRI of the macromolecular contrast agent albumin- 
gadolinium-DTPA was used to characterize the extravascular transport of macromolecules through the ECM of solid tumors in vivo (5). Macromolecular transport was characterized by imaging draining or pooling regions of the agent in the tumor. Increased draining areas were observed in the more invasive tumors that had a greater capacity for degrading the ECM (5). The contrast agent was rarely observed within the tumoral lymphatic vessels and most likely followed paths of least resistance through the ECM (5).

\section{IMAGING OF TUMOR VASCULATURE}

The angiogenic phenotype in cancers is usually associated with aggressiveness and increased metastasis. Tumors establish neovasculature from existing vasculature by secreting angiogenic factors (6) that induce endothelial cells to proliferate, invade, and migrate toward cancer cells, establishing the typically fragile, chaotic, and abnormal vasculature found in tumors (6). This abnormal vasculature results in areas of hypoxia, acidic extracellular $\mathrm{pH}$ partly due to poor perfusion, increased glycolysis, and substrate deprivation that result in radiation and drug resistance (7). MRI techniques using blood oxygen level-dependent contrast ( 8 ) or dynamic contrastenhanced MRI have been applied for several decades to characterize tumor vasculature (9), whereas MRS and MR spectroscopic imaging have been used to measure $\mathrm{pH}$ and metabolism (10).

Vascular properties may be imaged by dynamic contrastenhanced MRI in which MRI contrast agents are detected indirectly by their effects on water proton relaxation time constants (9). Low-molecular-weight gadolinium-based agents can be used to derive tracer kinetic parameters such as the volume transfer constant $\left(\mathrm{min}^{-1}\right)$ between the blood plasma and the extravascular extracellular space, the rate constant $\left(\mathrm{min}^{-1}\right)$ between the extravascular extracellular space and blood plasma, and the volume (\%) of the extravascular extracellular space per unit volume of tissue (9). Several lowmolecular-weight gadolinium-based agents such as gadoteridol (Prohance; Bracco Diagnostics, Inc.), gadopentetate dimeglumine (Magnevist; Bayer HealthCare Pharmaceuticals Inc.), gadoversetamide (OptiMARK; Covidien), and gadodiamide (Omniscan; Amersham Health) are available for clinical use.

A wider variety of contrast agents, including macromolecular agents, are available for characterizing tumor vasculature in preclinical studies. MRI of the macromolecular intravascular contrast agent albumin-gadolinium-DTPA has been used to characterize tumor vascular volume and the permeability surface area product (11). Recently, MRI of albumin-gadolinium-DTPA was combined with optical imaging to clarify the relationship between vascular parameters and hypoxia (11). These studies were performed using PC-3 human prostate cancer xenografts stably expressing enhanced green fluorescent protein under control of a hypoxia response element. Coregistered maps of vascular volume, permeability, and enhanced green fluorescent protein (Fig. 1A) demonstrated that hypoxic regions were characterized by low vascular volume, and frequently by high permeability because hypoxia increased the expression of vascular endothelial growth factor (VEGF), a potent permeability factor (11). Consistent with these observations, VEGF-overexpressing PC-3 tumors contained regions of high vascular volume that were also more permeable (Fig. 1B) (12). Control tumors exhibited few colocalized areas of high vascular volume and high permeability, whereas most of the vasculature in VEGF-overexpressing
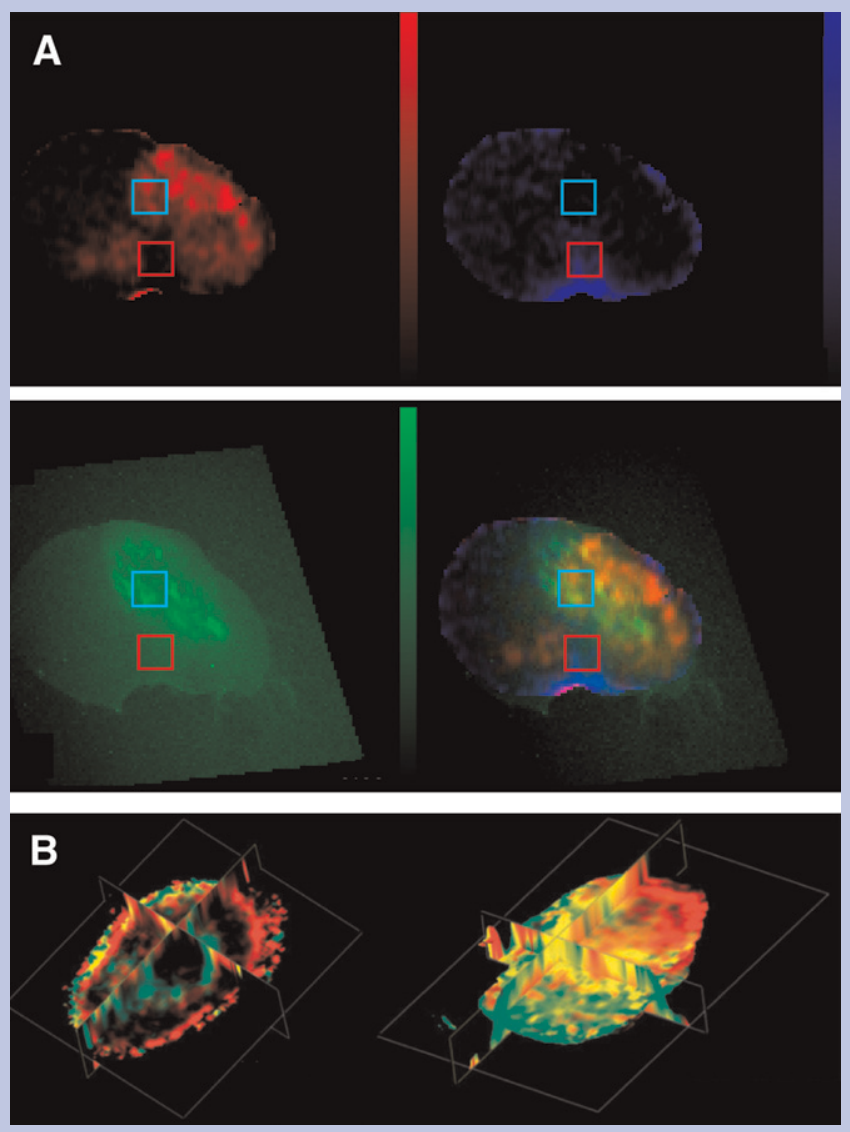

FIGURE 1. (A) In vivo permeability-surface area product map (scale, 0-12.36 $\mu \mathrm{L} / \mathrm{g} \cdot \mathrm{min}$ ) (top left); vascular volume map (scale, 0-214.37 $\mu \mathrm{L} / \mathrm{g}$ ) (top right); enhanced green fluorescent protein expression in excised tumor section acquired on optical microscope (scale, $0-118$ in gray-scale value) (bottom left); and composite image of the 3 previous maps, showing hypoxic regions with low vascular volume and high permeability in yellow and normoxic regions with low permeability and high vascular volume in blue (bottom right). (Adapted with permission from (11).) (B) Triplanar views of 3-dimensionally reconstructed fusion images of vascular volume (red) and permeability (green) from empty vector human $\mathrm{PC}-3$ prostate cancer xenograft model (left) and PC-3 prostate cancer xenograft model overexpressing VEGF (right). Control tumor showed distinct regions of red and green. In VEGF-overexpressing tumor, several regions exhibited yellow, demonstrating that regions of high vascular volume become permeable with VEGF overexpression. (Adapted with permission from (12). Copyright John Wiley \& Sons Limited.) 
tumors was highly permeable to albumin-gadolinium-DTPA (Fig. 1B).

\section{IMAGING HYPOXIA AND pH}

Oxygen partial pressure in tumors has been measured in vivo using both ${ }^{1} \mathrm{H}$ and ${ }^{19} \mathrm{~F}$ MRI $(13,14)$. Oxygen is sensed by reporter molecules selected for the oxygen dependence of their spin-lattice relaxation rate. The FREDOM technique (fluorocarbon relaxometry using echo planar imaging for dynamic oxygen mapping) was used to measure regional oxygen tension, with hexafluorobenzene as a reporter molecule (13). A ${ }^{1} \mathrm{H}$ MRS-detectable analog of hexafluorobenzene, hexamethyldisiloxane, which contains a single ${ }^{1} \mathrm{H}$ resonance with an oxygen partial-pressure-dependent $T_{1}$ value, was used to detect oxygen partial pressure in vivo after an intratumoral injection in prostate adenocarcinomas implanted in rats (14).

Tumoral $\mathrm{pH}$ influences tumor progression and efficacy of treatment (7). It has been shown that extracellular $\mathrm{pH}$ is usually lower in tumors than in normal tissues, which influences ECM degradation and thereby facilitates tumor progression and invasiveness (7). Several ${ }^{1} \mathrm{H}$ and ${ }^{31} \mathrm{P}$ MRS exogenous probes have been developed to measure extracellular $\mathrm{pH}(10) .{ }^{1} \mathrm{H}$ MRS probes with $\mathrm{pH}$-sensitive chemical shifts, such as imidazol-1-yl-3-ethyoxycarbonylpropionic acid, remain in the extracellular environment (10). The chemical shift of the $\mathrm{pH}$-sensitive signal can be detected by ${ }^{1} \mathrm{H}$ MR spectroscopic imaging and used to generate in vivo maps of tumor extracellular $\mathrm{pH}(10)$.

\section{CELL TRACKING}

MRI is also increasingly being used to noninvasively track the movement of stromal cells such as fibroblasts, immune cells, stem cells, or endothelial progenitor cells in tumors, by labeling these cells with MRI-detectable contrast agents $(15,16)$. Because these cells play a critical role in cancer progression and treatment, their visualization in vivo can be used to track their delivery and reveal new information on their function. Fibroblasts, a major constituent of connective tissue, play an important role in tumor progression and angiogenesis (15) and are involved in the synthesis of ECM components and formation of basement membrane. Recruitment of biotin-bovine serum albumin-gadolinium-DTPAlabeled fibroblasts into subcutaneous ovarian carcinoma tumors was tracked by MRI (15). Recruited labeled fibroblasts colocalized with the neovasculature at the tumor rim, suggesting a role for these stromal cells in tumor angiogenesis (15).

Bone marrow-derived endothelial precursor cells have also been shown to incorporate into tumor vasculature by differentiating into endothelium-like cells. Anderson et al. (16) labeled $\mathrm{Sca}^{+}$mouse bone marrow-derived cells enriched for endothelial precursor cells with dextran-coated superparamagnetic iron oxide nanoparticles to monitor their distribution in a mouse glioma model. Cells were visualized by a decreased signal intensity in $\mathrm{T}_{2}{ }^{*}$-weighted MR images (Fig. 2A), which revealed their incorporation into developing tumor vasculature located primarily at the periphery of the tumor (16).

The immune system is an important component of the TME, and cell-based therapies are increasingly being explored in cancer research. Tumor immunotherapy can be an efficient way to target and eliminate tumor cells (17). MRI monitoring of T-cell infiltration within tumors may be used to detect and improve T-cell homing. Tumor infiltration of activated $\mathrm{T}$ cells labeled with citrated anionic iron oxidebased nanoparticles was detected after intraperitoneal injection. Their infiltration correlated with tumor regression (17). Natural killer cells have also been tracked in vivo by MRI. Genetically engineered natural killer cells, directed against
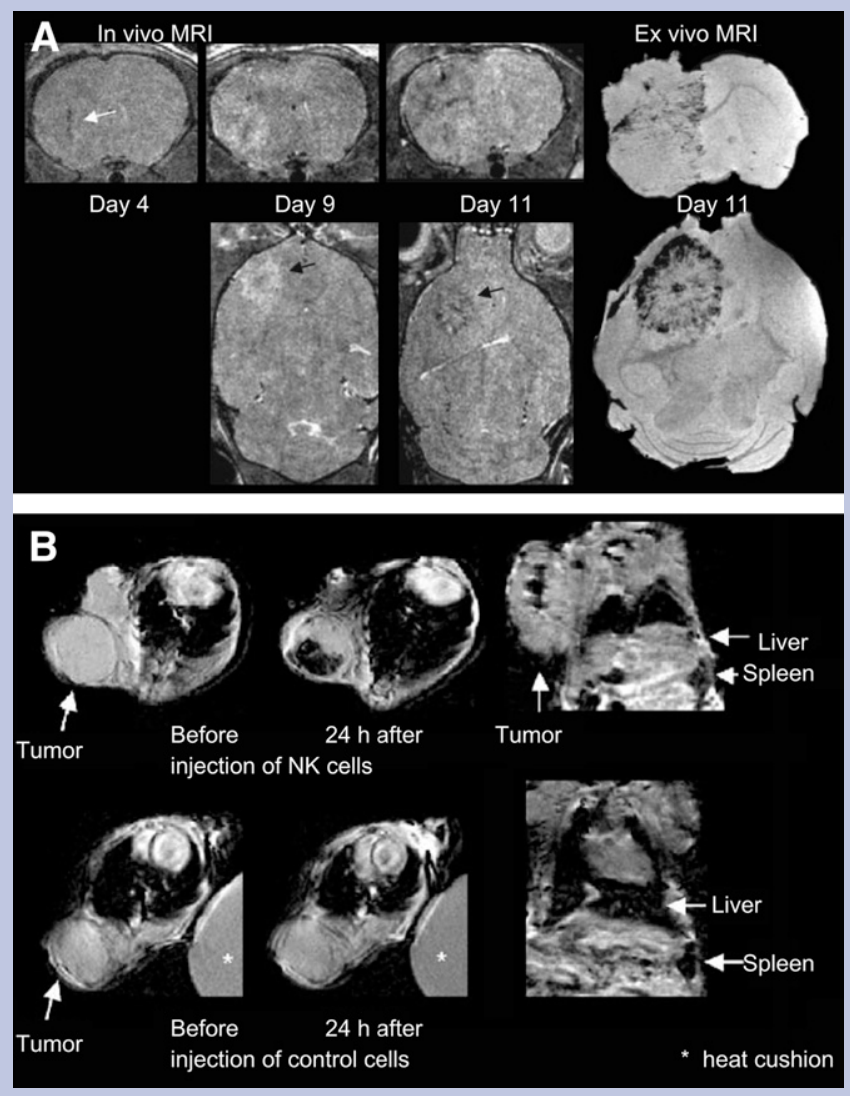

FIGURE 2. (A) On left are 3-dimensional RARE images acquired after injection of labeled Sca1+ bone marrow cells showing hypointense regions within and around tumor, because of incorporation of labeled cells into vascular structures or within parenchyma of tumors; on right is ex vivo gradientecho MR image at day 11. (Adapted with permission from (16). Research originally published in Blood [American Society of Hematology].) (B) At top are $\mathrm{T}_{2}{ }^{*}$-weighted images of HER2/neu ${ }^{+}$ NIH3T3 tumor (arrows) showing heterogeneous decline in signal intensity after injection of labeled NK cells directed against HER2/neu; at bottom, $\mathrm{T}_{2}{ }^{*}$-weighted images of HER2/neu ${ }^{+}$ NIH3T3 tumor (arrows) showing no change in signal intensity after injection of labeled nonspecific NK cells. (Adapted with kind permission of Springer Science and Business Media, from (18).) 
HER2/neu receptors and labeled with iron oxide-based contrast agents, have been injected in mice bearing a HER $2 /$ neu-positive mammary tumor (18). Figure 2B (top) shows the decrease in the MRI signal in $\mathrm{T}_{2}{ }^{*}$-weighted images of the tumor that resulted from the accumulation of targeted iron oxide-labeled cells, which were not visible with nonspecific labeled cells (bottom), demonstrating the specificity of targeting (18). Dendritic cells also play an important role in the initiation of immune response, because the injection of tumor-antigen-loaded dendritic cells can be used to enhance the endogenous immune response against tumor cells. de Vries et al. (19) have demonstrated the potential of MRI for tracking therapeutic cells in patients after intranodal injection of superparamagnetic iron oxide-labeled dendritic cells in melanoma patients (19).

\section{FUTURE DIRECTIONS}

Some exciting new areas for the future include targeting specific microenvironments or stromal compartments using image-guided incorporation of nano- and microdevices into tumors for slow release of therapeutic agents, gene delivery, and MR image-guided prodrug enzyme therapy as recently demonstrated by Li et al. (20). Small interfering RNA technology has provided a powerful means of targeting specific pathways. The use of image-guided delivery of small interfering RNA to downregulate specific pathways via targeted liposomes or other nanocarriers is an area that is already being explored (21) and can be exploited to target the TME. Major challenges for the future are the translation of preclinical applications for use in the clinic and the overcoming of limitations imposed by the inherent insensitivity of MRI detection, compared with radiopharmaceutical-based nuclear imaging. Gaining regulatory approval for MRI probes may prove difficult because these will not be used at tracer concentrations. The availability of preclinical and clinical multimodality imaging systems that incorporate the strengths and capabilities of MRI will increase the versatility of applications for understanding and targeting the TME and facilitate the clinical translation of these capabilities.

\section{Marie-France Penet Kristine Glunde Michael A. Jacobs Arvind P. Pathak Zaver M. Bhujwalla Johns Hopkins University School of Medicine Baltimore, Maryland}

\section{ACKNOWLEDGMENT}

Support from NIH P50 CA103175, R01 CA73850, R01 CA 82337, and R01 CA100184 is gratefully acknowledged.

\section{REFERENCES}

1. Mueller MM, Fusenig NE. Friends or foes: bipolar effects of the tumour stroma in cancer. Nat Rev Cancer. 2004;4:839-849.

2. Glunde K, Pathak AP, Bhujwalla ZM. Molecular-functional imaging of cancer: to image and imagine. Trends Mol Med. 2007;13:287-297.

3. Mazooz G, Mehlman T, Lai TS, et al. Development of magnetic resonance imaging contrast material for in vivo mapping of tissue transglutaminase activity. Cancer Res. 2005;65:1369-1375.

4. Shiftan L, Israely $\mathrm{T}$, Cohen $\mathrm{M}$, et al. Magnetic resonance imaging visualization of hyaluronidase in ovarian carcinoma. Cancer Res. 2005;65:1031610323.

5. Pathak AP, Artemov D, Neeman M, et al. Lymph node metastasis in breast cancer xenografts is associated with increased regions of extravascular drain, lymphatic vessel area, and invasive phenotype. Cancer Res. 2006;66:51515158

6. Folkman J. Angiogenesis: an organizing principle for drug discovery? Nat Rev Drug Discov. 2007;6:273-286.

7. Gatenby RA, Gillies RJ. Why do cancers have high aerobic glycolysis? Nat Rev Cancer. 2004;4:891-899.

8. Baudelet C, Cron GO, Gallez B. Determination of the maturity and functionality of tumor vasculature by MRI: correlation between BOLD-MRI and DCE-MRI using P792 in experimental fibrosarcoma tumors. Magn Reson Med. 2006;56: 1041-1049.

9. Laking GR, West C, Buckley DL, et al. Imaging vascular physiology to monitor cancer treatment. Crit Rev Oncol Hematol. 2006;58:95-113.

10. Pathak AP, Gimi B, Glunde K, et al. Molecular and functional imaging of cancer: advances in MRI and MRS. Methods Enzymol. 2004;386:3-60.

11. Raman V, Artemov D, Pathak AP, et al. Characterizing vascular parameters in hypoxic regions: a combined magnetic resonance and optical imaging study of a human prostate cancer model. Cancer Res. 2006;66:9929-9936.

12. Raman V, Pathak AP, Glunde K, et al. Magnetic resonance imaging and spectroscopy of transgenic models of cancer. NMR Biomed. 2007;20:186199.

13. Zhao D, Ran S, Constantinescu A, et al. Tumor oxygen dynamics: correlation of in vivo MRI with histological findings. Neoplasia. 2003;5:308-318.

14. Kodibagkar VD, Cui W, Merritt ME, et al. Novel ${ }^{1} \mathrm{H}$ NMR approach to quantitative tissue oximetry using hexamethyldisiloxane. Magn Reson Med. 2006;55:743-748.

15. Granot D, Addadi Y, Kalchenko V, et al. In vivo imaging of the systemic recruitment of fibroblasts to the angiogenic rim of ovarian carcinoma tumors. Cancer Res. 2007;67:9180-9189.

16. Anderson SA, Glod J, Arbab AS, et al. Noninvasive MR imaging of magnetically labeled stem cells to directly identify neovasculature in a glioma model. Blood. 2005; 105:420-425.

17. $\mathrm{Hu} \mathrm{DE}$, Kettunen MI, Brindle KM. Monitoring T-lymphocyte trafficking in tumors undergoing immune rejection. Magn Reson Med. 2005;54:14731479 .

18. Daldrup-Link HE, Meier R, Rudelius M, et al. In vivo tracking of genetically engineered, anti-HER2/neu directed natural killer cells to HER2/neu positive mammary tumors with magnetic resonance imaging. Eur Radiol. 2005;15: 4-13.

19. de Vries IJ, Lesterhuis WJ, Barentsz JO, et al. Magnetic resonance tracking of dendritic cells in melanoma patients for monitoring of cellular therapy. Nat Biotechnol. 2005;23:1407-1413.

20. Li C, Penet MF, Winnard P Jr, et al. Image-guided enzyme/prodrug cancer therapy. Clin Cancer Res. 2008;14:515-522.

21. Medarova Z, Pham W, Farrar C, et al. In vivo imaging of siRNA delivery and silencing in tumors. Nat Med. 2007;13:372-377. 